\title{
RAPID, METHOD FOR DETERMINATION OF METFORMIN AND CANAGLIFLOZIN IN PLASMA BY LIQUID CHROMATOGRAPHY-TANDEM MASS SPECTROMETER, APPLICATION TO BIOEQUIVALENCE STUDY
}

\author{
SAMBASIVA RAO PURAM ${ }^{1}$, NITHYA G ${ }^{2 *}$, SUBBA RAO NB ${ }^{1}$, RAMAN BATHEJA ${ }^{1}$ \\ ${ }^{1}$ Department of Bioanalytical, Vergo Clinicals, Corlim, Goa, India. ${ }^{2}$ Department of Chemistry, School of basic sciences, Vels University,
} Chennai, Tamil Nadu, India. Email: nithya.sbs@velsuniv.ac.in

Received: 10 August 2018, Revised and Accepted: 26 September 2018

\section{ABSTRACT}

Objective: The objective of the study was to develop and validate a rapid selective bioanalytical method for the simultaneous determination of metformin and canagliflozin in plasma by liquid chromatography-tandem mass spectrometer (LC-MS/MS) to facilitate bioequivalence study sample analysis. Stock solutions and spiking solutions were prepared accurately in methanol and $80 \%$ methanol in water, respectively.

Methods: Chromatography monitored using Analyst 1.6.2 software. Method was found selective, no matrix effect, reproducible and consistent recovery, accuracy, precision, and stable in aqueous as well as extracted/matrix samples. Method found linear over the range 10-2000 ng/ml for metformin and 30-6000 $\mathrm{ng} / \mathrm{ml}$ for canagliflozin. The method is successfully applied to analyze samples collected in a bioequivalence study after administration of metformin/canagliflozin $1000 / 150 \mathrm{mg}$ to 24 healthy male volunteers.

Results: Extraction was carried out using a simple solid phase extraction using mobile phase elution. $5 \mu$ sample delivered as injection volume in $1.000 \mathrm{ml} / \mathrm{min}$ isocratic mobile phase flow on turbo ion electron spray technique for positive mode on API $4000 \mathrm{MS}$.

Discussion: Chromatography achieved within 3 min using a mobile phase containing acetonitrile and $10 \mathrm{mM}$ ammonium formate buffer in a ratio of 70:30 on chromolith C18 analytical column. Q1/Q3 are 130.1/70.1, 136.3/77.1, 462.2/267.2 (with ammonium adduct), and 466.4/267.1 for metformin, metformin D6, canagliflozin, and canagliflozin D4, respectively.

Conclusion: Rapid, sensitive method for the simultaneous estimation of metformin and canagliflozin in plasma by LC-MS/MS is successfully developed, validated and applied to analyze 960 unknown samples of a bioequivalence study. Incurred sample reanalysis was revealed great reproducibility.

Keywords: Metformin+canagliflozin, Metformin and canagliflozin in plasma, Bioequivalence of metformin and canagliflozin, Canagliflozin+metformin by liquid chromatography-tandem mass spectrometer.

(c) 2019 The Authors. Published by Innovare Academic Sciences Pvt Ltd. This is an open access article under the CC BY license (http://creativecommons. org/licenses/by/4. 0/) DOI: http://dx.doi.org/10.22159/ajpcr.2019.v12i2.29027

\section{INTRODUCTION}

Metformin hydrochloride is chemically N, Ndimethylimidodicarbonimidic diamide hydrochloride $(1,1$ - dimethylbiguanide hydrochloride). Canagliflozin is chemically named as $(2 \mathrm{~S}, 3 \mathrm{R}, 4 \mathrm{R}, 5 \mathrm{~S}, 6 \mathrm{R})-2-[3-\{[5-(4-$ fluorophenyl)thiophen-2-yl]methyl\}-4-methylphenyl)-6-(hydroxymethyl) oxane-3,4,5-triol. Molecular weight of metformin is $129.2 \mathrm{~g} / \mathrm{mol}$ and molecular weight of canagliflozin is $444.5 \mathrm{~g} / \mathrm{mol}$ [1].

Metforminandcanagliflozinarefixeddosecombinationantihyperglycemic agents to improve glycemic control in diabetes patients; it has million dollars market worldwide. Metformin and Canagliflozin both are old drugs, and many formulations are available in the market along with fixed-dose combination formulations. When we checked at published methods, there are plenty of methods reported for the estimation of either metformin or canagliflozin in plasma by liquid chromatographytandem mass spectrometer (LC-MS/MS) or high-performance liquid chromatography (HPLC) or gas chromatography-MS, but no method is published for the simultaneous estimation in plasma by LC-MS/MS, development of single method is very difficult due to different polarities of drugs and other chemical properties. When we see at the structures of both, metformin ionizes in positive mode, whereas canagliflozin ionizes in negative mode. Devineni et al. reported method individual estimation methods for metformin and canagliflozin [2], Attimara et al. reported LC-MS method for the simultaneous determination of metformin and miglitol in human plasma: Application to pharmacokinetic studies [3], Kobuchi et al. reported method for canagliflozin by LC-MS/MS [4] and many others have reported either metformin in plasma or canagliflozin in plasma [5-21].

Simultaneous estimation one hand reduce the time of analysis to half so that the end results will be available early and regulatory submission can be done so early to capture the market share, and the other hand it reduces the cost of analysis significantly.

The objective of the current work is to develop, validate a simultaneous estimation method for the estimation of metformin and canagliflozin in human plasma by LC-MS/MS and its application to bioequivalence study.

\section{METHODS}

Metformin, metformin d6, canagliflozin, and canagliflozin D4 were purchased from Vivan life sciences and Clearsyth Labs Pvt. Ltd., Mumbai, India. HPLC grade solvents and chemicals were used during analysis, whereas the water is taken from the in-house water purification system. Hydrophobic lipophilic balance (HLB) $1 \mathrm{cc} / 30 \mathrm{mg}$ cartridges are from Phenominex, USA. LC-MS/MS system consists of ABSciex, API4000 with a front-end HPLC from Shimadzu. Analytical column is purchased from Merck Millipore, USA. A bioequivalence study was conducted based on a Independent Ethics Committee approved the protocol.

Stock solutions wereprepared by accurately weighingrespective working reference standard (STD) on balance, dissolved in methanol, labeled, 
and stored at refrigerated conditions. Working stock dilutions were made using 50\% methanol in water as diluent. Eight point calibration curve (CC) STDs are prepared in screened plasma for the CC range $10-2000 \mathrm{ng} / \mathrm{ml}$ for metformin and 30-6000 ng/ml for canagliflozin. Quality control (QC) samples at the lower limit of quantitation (LLOQ), lower QC, middle QC, and high QC levels are prepared at $102 \%$ to STD 1, 3 times to STD1, 45\% to STD8, and 76\% to STD8 in plasma. Required buffers, mobile phase, rinsing solutions are prepared as required and used up to 3 days. CC STDs and QC samples are bulk spiked and stored at $-70^{\circ} \mathrm{C}$ until analysis. Fresh CC STDs are prepared for the evaluation for Stability evaluation.

\section{Extraction procedure}

To a $0.200 \mathrm{ml}$ plasma sample, $0.050 \mathrm{ml}$ internal STD is added and vortexes for proper mixing; then the sample is diluted with $0.500 \mathrm{~mL}$ water to prevent clogging of cartridges; cartridges are conditioned with $1 \mathrm{ml}$ methanol followed by equilibration with $1 \mathrm{ml}$ water; above prepared sample is then loaded onto cartridge; cartridges are washed with $1 \mathrm{ml}$ water followed by $1 \mathrm{ml} \mathrm{10 \%}$ Acetonitrile in water; and then samples are eluted with $0.500 \mathrm{ml}$ mobile phase.

\section{Instrument conditions}

ABSCIEX API4000 Mass spectrometer coupled with Shimadzu LC system was utilized for reverse phase chromatography with an Isocretic flow at $1.000 \mathrm{~mL} / \mathrm{min}$ on RP18 Analytical column. Mass spectrometer conditions are presented under Table 1.

\section{Integration and quantitation}

Data acquired on Sciex analyst 1.6.2 software; automatic integration was done for a base to base and optimum integrations, CC is plotted with area ratio versus nominal concentrations of CC STDs, unknown sample concentrations are back-calculated from plotted curve using the formula $y=m x+c$, weighing factor was $1 / x^{2}$.

\section{RESULTS AND DISCUSSION}

\section{Method development}

About $100 \mathrm{ng} / \mathrm{ml}$ tuning solution in 90\% methanol was prepared from stock solution for analyte and internal STD; mass parameters were optimized using tuning solution, metformin ions found intense in positive mode and no ions found in negative mode, canagliflozin ions found intense in negative mode and no ions found in positive mode, but our objective is to get both in a single mode; hence, we decided to go for adduct form for canagliflozin as it can form ammonium adduct easily, adduction of canagliflozin found intense and reproducible. Labeled internal STDs are chosen as internal STDs to eliminate matrix effects and to get the highest reproducible results; matrix effects are nullified when labeled STDs are used. Based on the properties of the drugs correlating with ionization, the mobile phase, column conditions were optimized, metformin can easily extracted with protein precipitation technique but canagliflozin is difficult to extract; hence, we have tested for liquid-liquid extraction using polar to non-polar organic solvents but failed to good more than $50 \%$ recovery for both analytes, so finally we moved to solid phase extraction on HLB cartridges, and we got good recovery with reproducibility. Interference at retention times, carryover, matrix effect, precision, and accuracy tested and few alterations were done, and method is finalized. Pre-method validation was conducted before initiating method validation; this includes selectivity, carry over, precision, and accuracy, linearity, matrix effect, and bench-top stability. Weighing and regression method is finalized based on pre-method validation results.

\section{Method validation}

Method validation is conducted as per in-house STD operating procedure which is developed based on the European Medical Agency and United States Food and Drugs Administration guidelines on method validation. Autosampler carryover, selectivity, sensitivity, recovery, precision, accuracy, linearity, ruggedness for column, and analyst change, hemolysis and lipemic effect, aqueous, and extracted sample stability were evaluated. Method validation parameters, procedures, and acceptance are predefined.

\section{Sensitivity}

Sensitivity is performed to check the signal difference from baseline noise. LLOQ sample from 6 individual plasma lots was analyzed, signalto-noise ratio is calculated using software. Sensitivity found always $>5$. Limit of detection is not evaluated due to non-requirement in the current study.

\section{Selectivity}

Selectivity is performed to check the endogenous/exogenous interferences. Selectivity of the method is tested using 10 plasma lots including 6 normal, 2 hemolytic, and 3 hyperlipidemic by processing one blank, one blank+Internal STD, and one LLOQ sample from each individual lot. Percentage of interference at analyte/IS

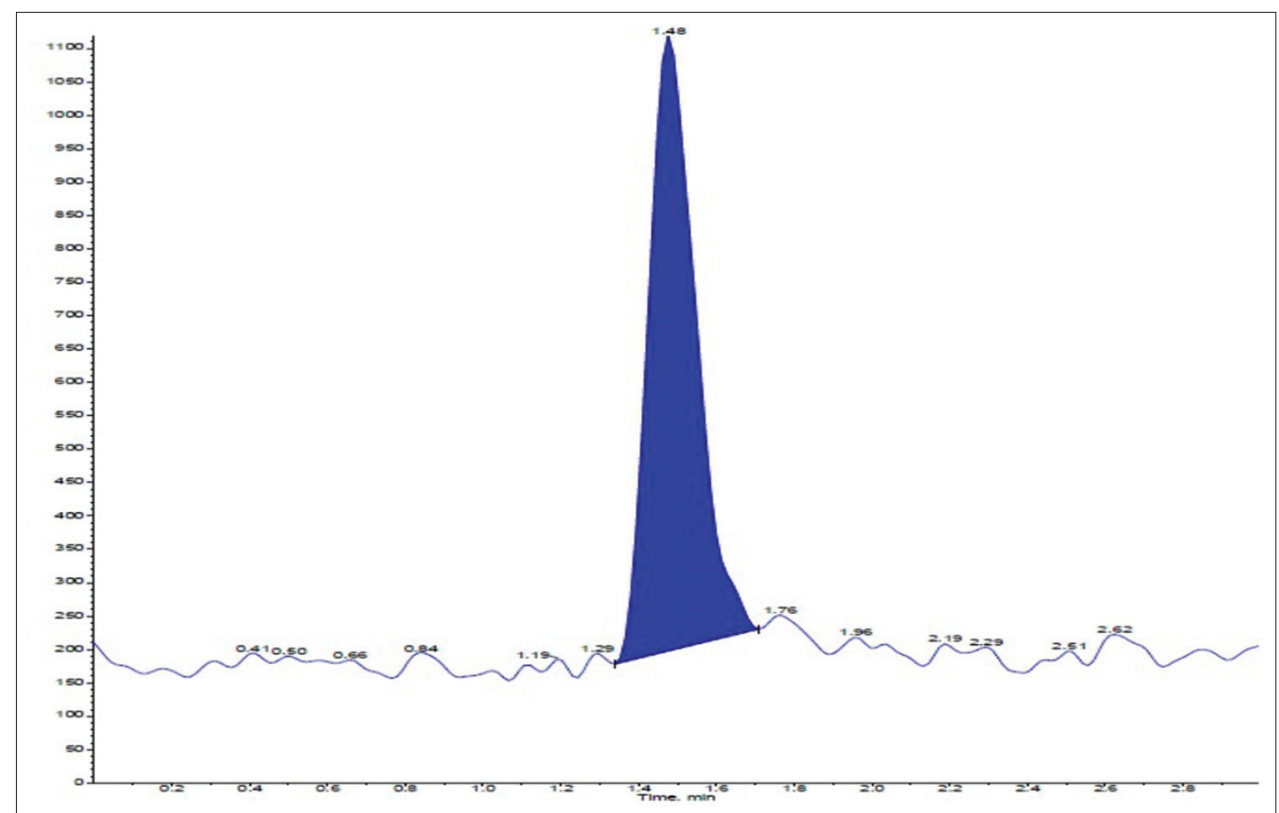

Fig. 1: Metformin lower limit of quantitation 


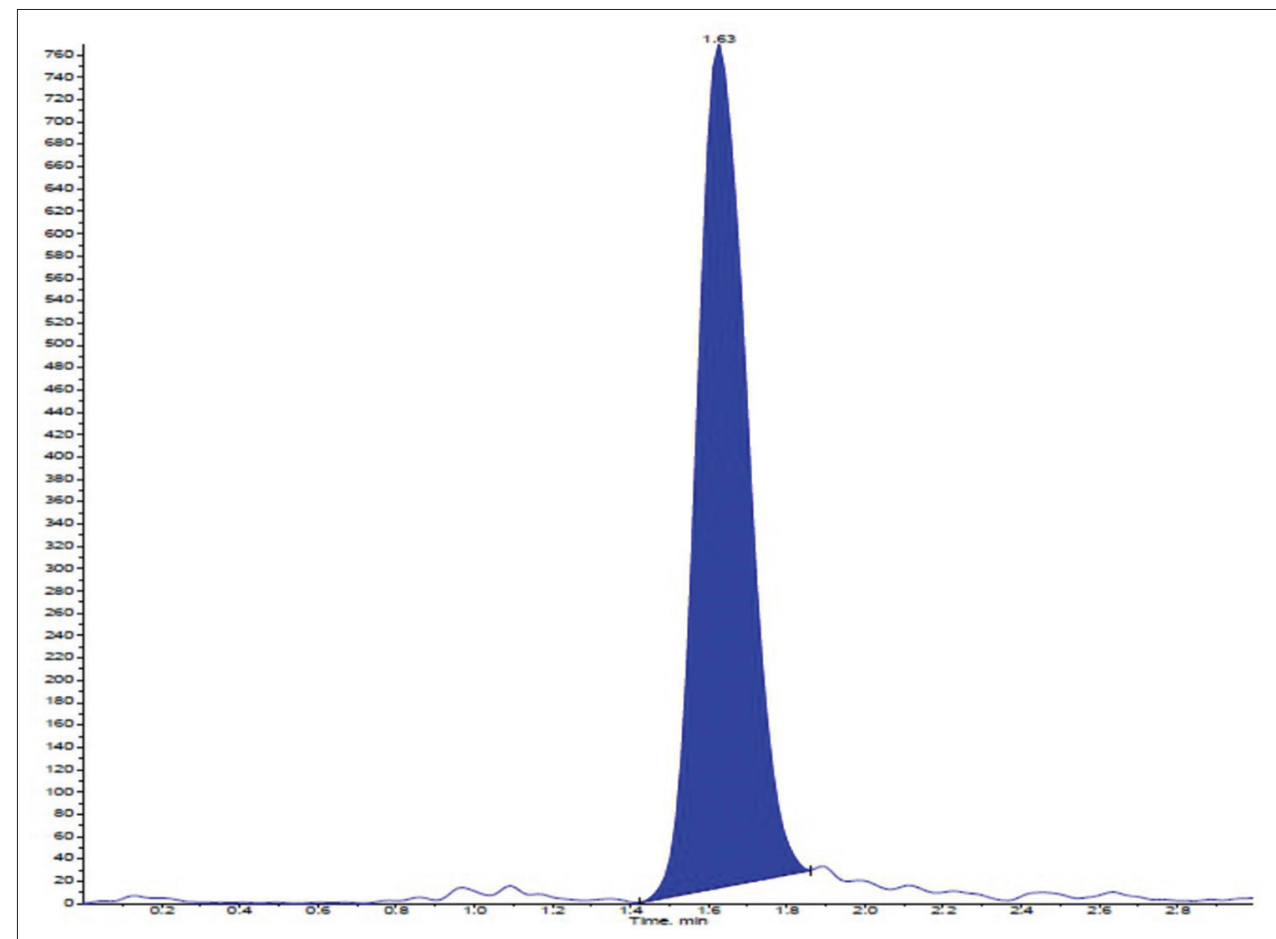

Fig. 2: Canagliflozin lower limit of quantitation

Table 1: Mass conditions

\begin{tabular}{|c|c|c|c|c|c|c|c|}
\hline Description & Q1 Mass & Q3 Mass & Dwell & DP & EP & CE & CXP \\
\hline Metformin & 130.100 & 71.100 & 200 & 60.00 & 10.00 & 20.00 & 13.00 \\
\hline Metformin $\mathrm{D}_{6}$ & 136.300 & 77.100 & 200 & 32.23 & 10.00 & 33.12 & 6.41 \\
\hline Canagliflozin (ammonium adduct) & 462.250 & 267.200 & 200 & 54.00 & 10.00 & 24.81 & 6.00 \\
\hline Canagliflozin $\mathrm{D}_{4}$ (ammonium adduct) & 466.400 & 267.100 & 200 & 60.00 & 10.00 & 25.00 & 15.00 \\
\hline Source temperature: $500^{\circ} \mathrm{C}$ & & \multicolumn{2}{|c|}{ IS voltage: $5000 \mathrm{kV}$} & \multicolumn{2}{|c|}{ Polarity: Positive } & \multicolumn{2}{|c|}{ Scan type: MRM } \\
\hline
\end{tabular}

DP: Declustering potential, EP: Entrance potential, CE: Collision energy, CXP: Collision exit potential

Table 2: Global statistics of precision and accuracy of metformin

\begin{tabular}{llll}
\hline \multicolumn{2}{l}{ Between-batch precision and accuracy } & & \\
\hline Description & LLOQQC & LQC & MQC \\
\hline Mean \pm SD & $10.1988 \pm 0.80233$ & $25.2631 \pm 1.07795$ & $807.3190 \pm 26.91706$ \\
Precision (\% CV) & 7.87 & 4.27 & 3.33 \\
Nominal concentration (ng/ml) & 10.533 & 26.492 & 817.656 \\
\% Accuracy & 96.83 & 95.36 & 98.74 \\
\hline
\end{tabular}

CV: Coefficient of variation, SD: Standard deviation, LLOQQC: Lower limit of quantitation quality control, LQC: Lower quality control, MQC: Middle-quality control, HQC: High-quality control

retention times is calculated considering LLOQ area 100 . Selectivity found acceptable. Sample LLOQ chromatograms are presented under Figs. 1 and 2.

\section{Recovery}

Recovery of extraction efficiency was tested. Absolute recovery using three levels of QC s was tested. QC samples are processed and injected along with equivalent pure samples prepared in reconstitution solution; mean areas at each QC level are compared. Recovery found $>70 \%$ with consistent and reproducible.

\section{Linearity, precision, and accuracy}

Precision and accuracy batch consists of one cc and $24 \mathrm{qC}$ samples at four different levels were analyzed. A total of 4 precision and accuracy batches were analyzed in two different days for intra- and inter-day calculations. CC for both analytes found linear all the times with $r>0.99$. Global statistics are tabulated in Tables 2 and 3.

\section{Ruggedness}

Ruggedness of the method is tested for the changes column, analyst, and instrument. One precision and accuracy were analyzed by a different analyst on different column/different instrument. Results found acceptable. Precision found $2-7 \%$ whereas accuracy found between $86 \%$ and $109 \%$

\section{Stability}

Stability of analytes and internal STDs is tested in aqueous solutions for short-term as well as long-term by injecting a sample from stability solution along with comparison solution prepared freshly. Stability of processed samples for different storage conditions such as evaporation stability, wet extract stability, autosampler stability, and wet extract bench top stability was done along with the stability of drugs in plasma at different conditions such as benchtop stability, freeze-thaw stability, whole blood stability, and long-term stability. Stock solution found stable up to $6 \mathrm{~h}$; benchtop stability found acceptable for $6 \mathrm{~h}$, freeze- 
Table 3: Global statistics of precision and accuracy of canagliflozin

\begin{tabular}{lllll}
\hline \multicolumn{2}{l}{ Between-batch precision and accuracy } & & & \\
\hline Description & LLOQQC & LQC & MQC & HQC \\
\hline Mean \pm SD & $29.9213 \pm 1.30406$ & $74.6727 \pm 2.19700$ & $2405.5809 \pm 89.89150$ & $4702.9788 \pm 165.34826$ \\
Precision (\% CV) & 4.36 & 2.94 & 3.74 & 3.52 \\
Nominal concentration (ng/ml) & 31.187 & 78.437 & 2420.894 & 4637.728 \\
\% Accuracy & 95.94 & 95.20 & 99.37 & 101.41 \\
\hline
\end{tabular}

CV: Coefficient of variation, SD: Standard deviation, LLOQQC: Lower limit of quantitation quality control, LQC: Lower quality control, MQC: Middle-quality control, HQC: High-quality control

thaw stability found stable up to IV cycles, autosampler stability found stable up to $52 \mathrm{~h}$, whole blood stability found acceptable for $2 \mathrm{~h}$, and long-term stability in plasma found stable for 28 days.

Miscellaneous experiments such as dilution integrity, hemolysis effect, lipemic effect, selectivity in the presence of concomitant drugs were conducted and found all experiments acceptable.

\section{Application of the method}

Validated method has been applied to a bioequivalence study involved 24 volunteers, fixed-dose combination formulation of metformin and canagliflozin was administered as per independent Ethics Committee approved protocol to 24 volunteers in a two way crossover design with 7 days washout period, volunteers whoever meeting inclusion and exclusion criteria of protocol and whoever given informed consent were recruited after successful completion of screening tests. Dosing was done under fasting conditions, regular blood sampling was done, vitals checked, blood samples were centrifuged, and plasma was separated then stored at $-70^{\circ} \mathrm{C}$ until sample transfer to the bioanalytical department for analysis. No adverse events were reported, and all 24 volunteers completed the clinical phase of the study. All samples of a given subject were analyzed using 8 point CC and interspersing QC samples. Batch evaluation was conducted and samples selected for repeat analysis for analytical reasons, no batch failures found, 22 individual samples were reanalyzed for an improper internal STD response. Incurred sample reanalysis (ISR) was conducted for 10\% samples, $95 \%$ samples ISR values are matching with original assay value. After completion of the analysis, quality assurance department reviewed that subject concentration data are submitted to biostatistician in a locked Microsoft Excel file. Statistician conducted pharmacokinetic analysis on win online, and biostatistician analysis is on SAS software. Non-compartmental analysis is done to check $90 \%$ confidence intervals for bioequivalence review. 90\% confidence intervals found between $80 \%$ and $125 \%$ for a test when compared to reference formulation. Mean $\mathrm{C}_{\max }$ found is $1256 \mathrm{ng} / \mathrm{ml}$ for metformin and $390 \mathrm{ng} / \mathrm{ml}$ for canagliflozin.

\section{CONCLUSION}

Rapid, sensitive method for the simultaneous estimation of metformin and canagliflozin in plasma by LC-MS/MS is successfully developed, validated and applied to bioequivalence study sample analysis. Chromatography achieved on the C18 column on acetonitrile, ammonium formate mobile phase, extraction was conducted using solid phase extraction on HLB cartridges. Positive MRM detection was performed on API 4000 LC-MS/MS. The analysis is rapid with 3 min run time for each sample, around 200 samples can be analyzed daily, the method found selective from endogenous/exogenous interferences; all validation parameters proved that the method is accurate, precise, and stable. Method found linear over the range $10-2000 \mathrm{ng} / \mathrm{ml}$ for metformin and $30-6000 \mathrm{ng} / \mathrm{ml}$ for canagliflozin. The method is applied to analyze 960 unknown samples of a bioequivalence study after administration of a fixed-dose combination of metformin and canagliflozin tablets. ISR was revealed great reproducibility with over $90 \%$ ISR sample concentrations matching with original assay concentrations.

\section{ACKNOWLEDGMENT}

We truly thankful to the Vergo Management for accepting to do this work and we also thankful to Guide Dr. Nitya for her valuable guidance.

\section{AUTHORS' CONTRIBUTIONS}

The research work has been jointly carried out by the authors under the guidance of Dr. G. Nithya, the Corresponding author.

\section{CONFLICTS OF INTEREST}

Nil.

\section{REFERENCES}

1. Available from: http://www.rxlist.com/invokamet-drug/clinicalpharmacology.htm. [Last accessed on 2017 Apr 10].

2. Devineni D, Curtin CR, Ariyawansa J, Weiner S, Stieltjes H, Vaccaro $\mathrm{N}$, et al. Bioequivalence of canagliflozin/metformin immediate release fixed dose combination tablets compared with concomitant administration of single components of canagliflozin and metformin in healthy fed participants. J Bioequiv Availab 2014;6:164-73.

3. Attimara MV, Harsha NS, Nair AB, Aldhubaib BE, Alhaider IA. LCMS Method for the Simultaneous Determination of Metformin and Miglitol in Human Plasma: Application to Pharmacokinetic Studies. Available from: http://www.msacl.org/2015.

4. Kobuchi S, Yano K, Ito Y, Sakaeda T. A validated LC-MS/MS method for the determination of canagliflozin, a sodium-glucose co-transporter 2 (SGLT-2) inhibitor, in a lower volume of rat plasma: Application to pharmacokinetic studies in rats. Biomed Chromatogr 2016;30:1549-55.

5. Madhukar A, Prince A, Kumar R, Sanjeeva Y, Jagadeeshwar K, Raghupratap D. Simple and sensitive analytical method development and validation of metformin hydrochloride by RP-HPLC. Int J Pharm Pharm Sci 2011;3:3.

6. Gaware D, Patil RN, Harole M. A validated stability indicating RP-HPLC method for simultaneous determination of metformin and canagliflozin in pharmaceutical formulation. World J Pharm Pharm Sci 2015;4:631-40.

7. Pandya RH, Rathod R, Maheswari DG. Bioanalytical method development and validation for simultaneous determination of linagliptin and metformin drugs in human plasma by rp-hplc method. Pharmacophore 2014;5:202-18

8. Devineni D, Curtin CR, Polidori D, Gutierrez MJ, Murphy J, Rusch S, et al. Pharmacokinetics and pharmacodynamics of canagliflozin, a sodium glucose co-transporter 2 inhibitor, in subjects with Type 2 diabetes mellitus. J Clin Pharmacol 2013;53:601-10.

9. Gaikwad A, Gavali S, Narendiran AS. A simple and sensitive method for determination of metformin and sitagliptin in human plasma using liquid chromatography and tandem mass spectrometry. Int J Pharm Pharm Sci 2013;5:463-70

10. Polagani SR, Pilli NR, Gajula R, Gandu V. Simultaneous determination of atorvastatin, metformin and glimepiride in human plasma by LC-MS/MS and its application to a human pharmacokinetic study. J Pharm Anal 2013;3:9-19.

11. Heinig K, Bucheli F. Fast liquid chromatographic-tandem mass spectrometric (LC-MS-MS) determination of metformin in plasma samples. J Pharm Biomed Anal 2004;34:1005-11.

12. Jian W, Ying-Wu WG, Jing-Kai G. Determination of metformin in human plasma by liquid chromatography-tandem mass spectrometric assay. Chem Res Chin Univ 2005;21:246-50.

13. Zhanga W, Hana E, Zhaoa H. Determination of Metformin in Rat Plasma by HILIC-MS/MS Combined with Tecan Automation and Direct Injection. Available from: http://www.wileyonlinelibrary.com/bmc.2673. 
14. Iqbal M, Khalil NY, Alanazia AM, Al-Rasod KA. A simple and sensitive high performance liquid chromatography assay with a fluorescence detector for determination of canagliflozin in human plasma. Anal Methods 2015;7:45-7.

15. Chhetri HP, Thapa P, Van Schepdael A. Simple HPLC-UV method for the quantification of metformin in human plasma with one step protein precipitation. Saudi Pharm J 2014;22:483-7.

16. Uçaktürk E. The development and validation of a gas chromatographymass spectrometry method for the determination of metformin in human plasma. Anal Methods 2013;2013:18

17. Duttaa P, Rayb S, Nagarajanc K. Docking study of some glutamic acid derivatives as potent antineoplastic agents. Int J Pharm Pharm Sci 2014;6:419-22.
18. Sharma RB, Chetia D. Docking studies on quinine analogs for plasmepsin-II of malaria parasite using bioinformatics tools. Int J Pharm Pharm Sci 2013;5:681-5.

19. Swetha A, Kuber BR. A novel stability-indicating reverse phase liquid chromatographic method for the simultaneous estimation of metformin and teneligliptin in pure and pharmaceutical formulations. Int J Appl Pharm 2018;10:274-80.

20. Das D, Chakraborty J, Dash S. Bioequivalence study of antidiabetic activity between two marketed formulations of metformin on glucocorticoid-induced hyperglycemia in rabbit. Int J Curr Pharm Res 2017;9:47-50

21. Kulkarni AA, Vaidya IS. Flow injection analysis: An overview. J Crit Rev 2015;2:19-24. 